\title{
Relations between ejection fraction and ventricular volume, and their alteration by chronic beta-blockade
}

\author{
P L M KERKHOF, J BAAN, B BUIS, A C ARNTZENIUS \\ From the Departments of Paediatrics and Cardiology, Leiden University Hospital, Leiden, The Netherlands
}

SUMMARY This study describes the newly discovered relation between ejection fraction and endsystolic volume index (ESVI) of the left ventricle as obtained by angiography at the time of cardiac catheterisation. Linear regression analysis shows that ejection fraction $(\%)=82 \cdot 0-0.62 \mathrm{ESVI}\left(\mathrm{ml} / \mathrm{m}^{2}\right)$ but the correlation for patients receiving beta-adrenergic blocking drugs is significantly lower compared with the untreated group. Non-linear analysis, applied to cover also the asymptotic range for ejection fraction $<20 \%$, shows similar results. The good relation between the two indices indicates that the index ejection fraction may derive its clinical importance directly from the more fundamental index end-systolic volume index by virtue of the operation of the beta-adrenergic system on the heart.

Generally, ejection fraction has been accepted clinically as a useful index to assess ventricular performance. ${ }^{1}$ To date, no basis other than empirical observations has been provided on which the importance ${ }^{2}$ of ejection fraction is founded. Moreover, some investigators have questioned seriously the relevance of ejection fraction as a unique discriminator of ventricular performance. ${ }^{34}$ End-diastolic volume index (EDVI) has been regarded as a primary determinant of ventricular function, mainly in conjunction with the Frank-Starling law of the heart. Several studies have shown a more or less hyperbolic relation between ejection fraction and enddiastolic volume index. ${ }^{5-7}$ Recently, however, considerable attention has been given to systolic events during the cardiac cycle, and particularly to the endsystolic volume index ${ }^{89}$ and related quantities such as systolic elastance. ${ }^{10-12}$ Since end-systolic volume is the volume to which the heart is able to contract under given conditions of preload and afterload this index is just as fundamental as end-diastolic volume to judge cardiac performance. Consequently, it seems logical to investigate the relation between the two systolic indices, ejection fraction and end-systolic volume index (ESVI). The present study documents a remarkable relation between the empirical index ejection fraction and the functional variable end-systolic volume index in a large group of patients. In addition, the effect of adrenergic blockade on this relation is studied by way of analysis of a separate group of patients under chronic treatment with beta-blockade.

Received for publication 12 November 1980

\section{Patients and methods}

The population under consideration consisted of 165 unselected patients having angina and admitted to our clinic for medical examination. Their suitability for coronary bypass surgery was evaluated by selective angiocardiography, employing the Judkins technique. Fifty-two patients were not receiving any sympatholytic medication, while all the others (113) continued to receive their usual doses of beta-adrenergic blocking agents during the period of diagnostic catheterisation. Left ventricular cavity volume was determined angiographically using the right anterior oblique projection and the area-length method of Dodge, ${ }^{13}$ in combination with correction terms, derived from studies by Lange and coworkers, ${ }^{14}$ yielding $V_{\text {true }}=0.72 V_{\text {measured }}$ $-4.7 \mathrm{ml}$. Volumes were normalised for body surface area to obtain end-systolic and end-diastolic volume indices, expressed as $\mathrm{ml} / \mathrm{m}^{2}$. Ejection fraction (EF) was calculated using the definition formula $E F=1-E V S I /$ EDVI. Details of the methods used have been extensively described previously. ${ }^{9}$ Briefly, standard linear and non-linear regression methods were applied to describe the correlation of ejection fraction to endsystolic volume index and end-diastolic volume index. Basically, simple linear regression analysis may be applied to these data, as long as ejection fraction is not within the asymptotic region below $20 \%$. As documented previously,' asymptotic behaviour may be present for data points in the latter region. On the basis of theoretical considerations, we predicted ${ }^{9}$ that the relation between ejection fraction and end-systolic 


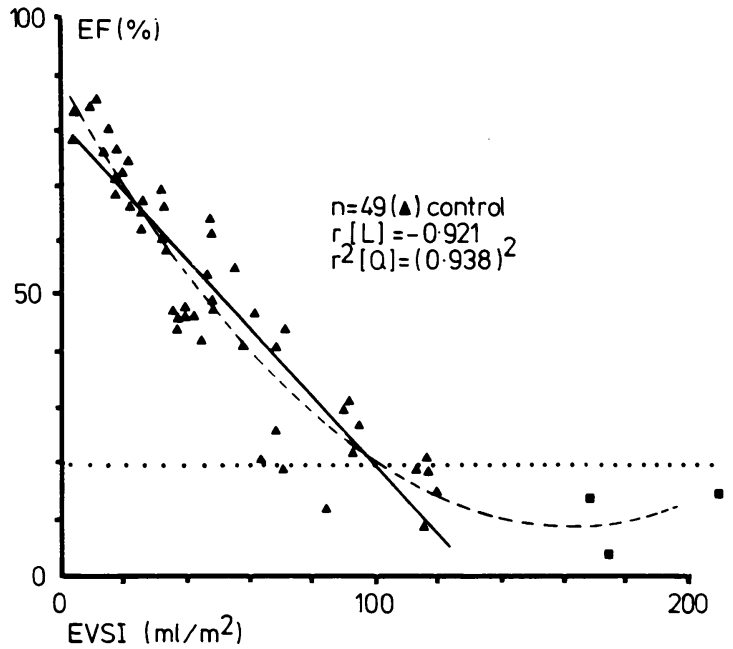

Fig. 1 Relation between ejection fraction (EF) and end-systolic volume index (ESVI) yields a high linear correlation coefficient $r$ [L] for 49 patients (triangles) not using beta-adrenergic blockers. $r[L]$ is not significantly different from $r^{2}[Q]$, obtained from quadratic analysis. The three squares refer to additional data points, positioned in the asymptotic region (for definition, see text). The linear regression line for $n=49$ (triangles) and the quadratic regression curve for all data points $(n=52)$ in the control group (broken curve) are shown.

volume index was quasi-hyperbolic, that is in abbreviated form: EF $\alpha \mathrm{ESVI} /(\mathrm{ESVI}-\mathrm{c})$, where $\mathrm{c}$ is a constant.

The asymptotic region is defined here as that area below the ejection fraction $=20 \%$ line where, in addition, end-systolic volume index values are beyond the range determined by the 2 SEE (twice standard error of the estimate) line calculated on the basis of the regression line obtained by pooling all data points having ejection fractions of more than $20 \%$. Differences between regression coefficients were analysed using the Student $t$ distribution, and the significance of different $r$ values was tested using the Fisher $\mathrm{z}$-transformation.

\section{Results}

Ejection fraction and end-systolic volume index values for both patient groups are shown in Fig. 1 and 2, along with their linearised and quadratic regression curves. In our study, 156 patients had an ejection fraction greater than $20 \%$, thus yielding a 2 SEE line given by

$$
\mathrm{EF}(\%)=97.5-0.66 \mathrm{ESVI}\left(\mathrm{ml} / \mathrm{m}^{2}\right)
$$

This procedure gives three deviating data points in our study as marked by the square symbols in Fig. 1, indicating that over a wide range of values of endsystolic volume values the relation between ejection

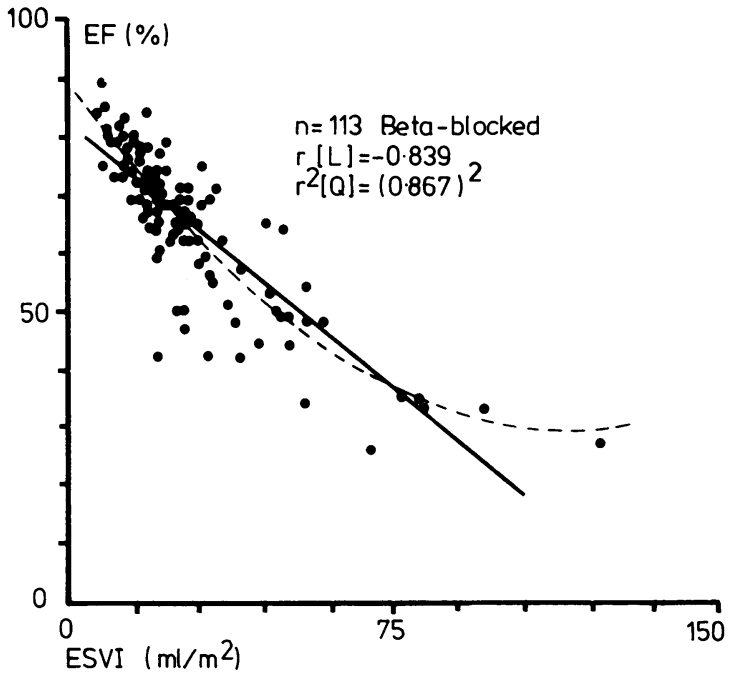

Fig. 2 Relation between EF and ESVI similar to that in Fig. 1, but now for the patient group $(n=113)$ under chronic beta-blockade. Both the linear (solid line) and the quadratic regression (broken line) curves are shown. Both correlation coefficients $r[L]$ and $r^{2}[Q]$ are significantly reduced $(p<0.02)$ compared with the untreated group. Note the different scale of the abscissa, compared with Fig. 1.

fraction and end-systolic volume index may be fairly well approximated by a linearised approach, yielding an overall linear correlation coefficient of -0.899 (Table) for 162 patients, while neglecting three data points (squares in Fig. 1) in the asymptotic region. It should be noted that in this asymptotic region the variable ejection fraction is about equal for patients with widely varying end-systolic and end-diastolic volume indices, which may imply that in these cases the ejection fraction is not an unambiguous index of left ventricular function. To allow for aligned comparison of the two groups, we decided to discard these three points, and to concentrate primarily on the linearised region of the ejection fraction vs. end-systolic volume index curves. After these patients were subdivided into groups, namely those untreated and those treated with beta-blocking agents, the following results were obtained:

$$
\begin{array}{ll}
49 \text { untreated: } & E F(\%)=80.3-0.60 \text { ESVI with } \\
& r=-0.921 \text { and } p<0.0005 \\
113 \text { beta-blocked: } & E F(\%)=82 \cdot 1-0.61 \text { ESVI with } \\
r=-0.839 \text { and } p<0.0005
\end{array}
$$

The slight differences of the regression coefficients (that is the slopes and intercepts of the lines) are far from significant. The two groups, however, generate correlation coefficients $r$, which are significantly $(p<0.02)$ 


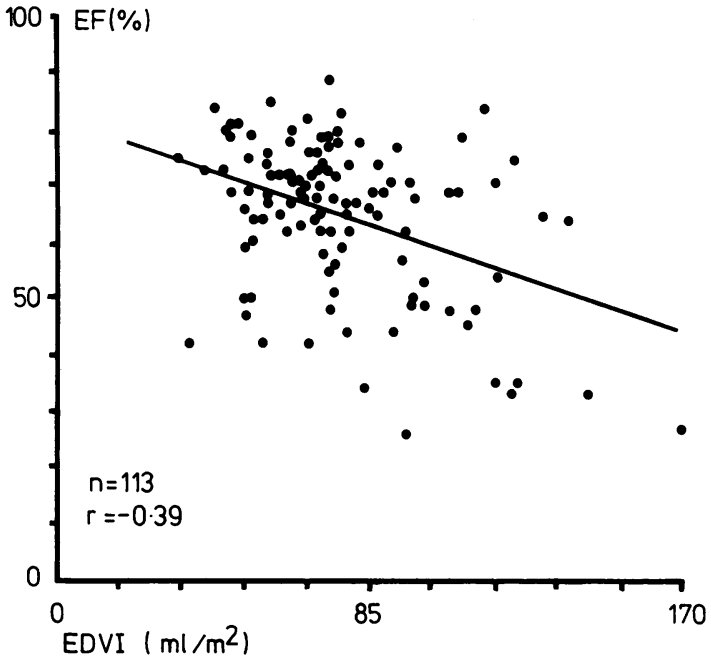

Fig. 3 Any type of relation between ejection fraction $(E F)$ and end-diastolic volume index (EDVI) is virtually absent. Data points refer to the group of patients under chronic betablockade.

different. This observation indicates that the unique relation between the ejection fraction and end-systolic volume index is lost to a certain extent in patients treated with beta-adrenergic blockers.

If the procedure of omitting the three asymptotic points is abandoned, it is necessary to apply non-linear regression analysis. Quadratic analysis confirms that ejection fraction and end-systolic volume indexes are again excellently correlated, and also that the correlation coefficient is reduced for the group of patients under chronic beta-blockade. The difference of the

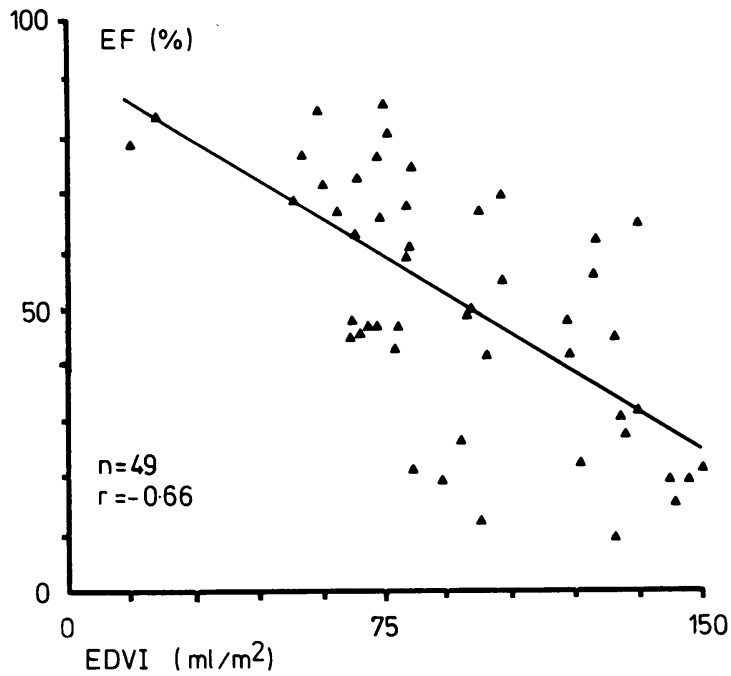

Fig. 4 A poor correlation between $E F$ and EDVI is also found for the group of patients not using beta-blockers, in contrast to results obtained from analysis of EF vs. ESVI, as shown in Fig. 1.

correlation coefficients is similarly significant $(\mathrm{p}<0.002)$.

When ejection fraction is plotted as a function of end-diastolic volume index for our patients, a poor linear correlation is found. The $r$ value is only -0.56 when all 162 patients are considered (Table), while $r=-0.39$ (Fig. 3) for the treated and $r=-0.66$ (Fig. 4) for the untreated groups, respectively. Not only was a high correlation absent, but in addition it was impossible to recognise a hyperbolic or any other nonlinear relation for these collections of data points. The

Table Survey of correlations between EF and ESVI as well as EDVI, for both patient groups separately and combined

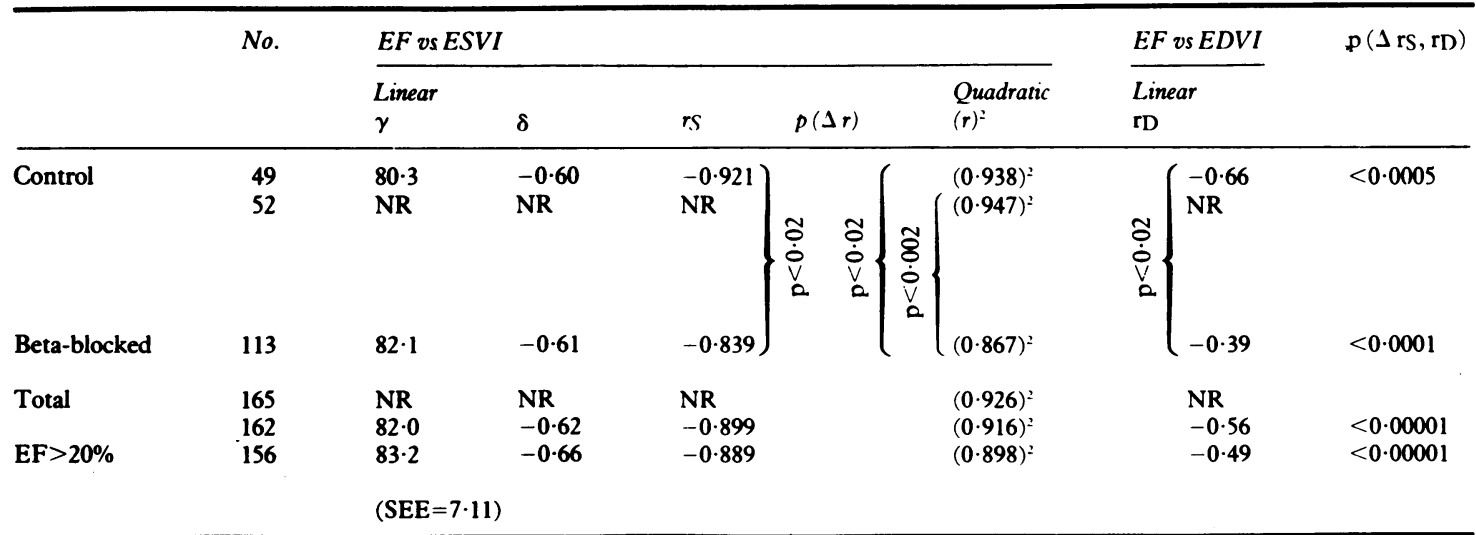

Abbreviations: No., number of patients analysed; rs, linear correlation coefficient for EF vs ESVI; rD, linear correlation coefficient for EF vs EDVI; $\mathrm{p}(\Delta \mathrm{rS}, \mathrm{rD})$, significance of difference between $\mathrm{rS}$ and $\mathrm{rD} ; \mathrm{p}(\Delta \mathrm{r})$, significance of difference between $\mathrm{r}$ values for control against treated group; $\gamma$, intercept (\%) of linear EF vs ESVI; $\delta$, slope $\left(\mathrm{m}^{2} / \mathrm{ml}\right)$ of linear EF $v s$ ESVI. NR: not relevant (see text). SEE=standard error of the estimate of the regression line. 
correlation coefficient is again significantly $(p<0.02)$ reduced after beta-blockade.

\section{Discussion}

Regression analysis applied to volumetric data from our patients discloses that ejection fraction and endsystolic volume index are highly correlated. For the range of ejection fraction values of main interest (that is above about $20 \%$ ) the relation can be fairly well approximated by a linearised description. A quadratic approach turned out to be necessary in order to take into account the asymptotic part at the higher endsystolic volume index region. To our knowledge, no previous investigators have discovered this remarkable relation. Analysis9 of data collected from studies by other investigators strikingly confirmed our observations with respect to the excellent relation between ejection fraction and end-systolic volume index.

In addition, the effects of beta-blocking agents on this relation were investigated. Most studies of betaadrenergic blockade are concerned with effects on heart rate, cardiac output, and blood pressure, while only limited information with respect to the effects on left ventricular volume is available. We were unable to find published data providing sufficient information to allow for due statistical comparison of groups of patients with and without beta-adrenergic blockade. Therefore, our study was designed to cover numbers of patients in both groups which enabled firm statistical statements with respect to different $r$ values for regression analysis applied to both groups. Ideally, this type of research should be carried out on one single group, serving as its own control. Since we are considering chronic effects of beta-blockade, such an investigation would not be technically feasible. When both our groups are considered separately, it appears that the linear regression coefficients (that is intercept $\gamma$ and slope $\delta$ ) are virtually the same. The correlation, however, is significantly higher for the untreated patients compared with the group receiving betablockers when linear regression as well as quadratic regression analysis is carried out. This indicates that end-systolic volume index and ejection fraction are no longer of equal importance when evaluating cardiac performance after beta-blockade. Further investigations, for example on dose dependent effects, may elucidate important implications for the analysis of left ventricular performance.

The purpose of this paper is to show the simple connection between the two indices, which contrasts the weak relation between ejection fraction and enddiastolic volume index, rather than applying optimal curve fitting procedures, or describing analytically the precise relation between ejection fraction and endsystolic volume index. A linearised approach un- doubtedly has advantages over more complicated descriptions. It appears that the relation between ejection fraction and end-systolic volume index (as exemplified by the high linear correlation coefficients found, which do not differ significantly from those obtained from non-linear regression analysis) is almost perfectly linear over a substantial range. Deviations occur mainly at the extreme regions where the ejection fraction exceeds $85 \%$ and is below about $20 \%$, where the relation appears to possess curvilinear portions. The linear regression coefficients themselves are more representative than the quadratic coefficients, because they are individually less subject to variability of just a few data points at the boundaries, especially when a considerable linear range is visually evident. Even higher order equations may be required to accommodate a smooth asymptotic fit at the higher endsystolic volume index range. In fact, a Taylor series expansion of the expression ESVI/(ESVI-c), which was predicted in an earlier theoretical study, ${ }^{9}$ already suggests possible involvement of a higher order polynomial. But again, curve fitting was not our aim in this study. For clarity and convenience, a linearised approach can be considered, thereby simplifying interpretation and comparison of experimental results. We did pursue quadratic analysis primarily in order to incorporate data points in the asymptotic region, as defined above. We were able, however, both for linear as well as for quadratic analysis, to prove that the correlation coefficients are significantly reduced by changes induced by the chronic administration of sympatholytic drugs in patients with angina pectoris.

The analysis of our patient data reported here indicates that the ejection fraction and end-diastolic volume index are poorly correlated, and do not exhibit a hyperbola-like relation. This finding indicates that the relation between the ejection fraction and enddiastolic volume index is not necessarily hyperbolic as claimed by some investigators, ${ }^{5-7}$ while denied by others. ${ }^{15}$ In addition, the ejection fraction is far better $(p<0.0005)$ related to end-systolic volume index than to end-diastolic volume index (see Table). For purposes of comparison it is essential to consider identical measurement techniques. ${ }^{9}$ Assumptions concerning geometry of the cavity certainly will affect ${ }^{2}$ the estimates of end-systolic and end-diastolic volume indexes. Thus, the precise values of the regression coefficients may depend on the particular technique employed to determine left ventricular volume. Notwithstanding the variety of methods used by various investigators, we ${ }^{9}$ always found an excellent correlation between the ejection fraction and end-systolic volume index. This study is unique with respect to the detection of a particular effect of adrenergic control on left ventricular volume in man. The simple relation established in this study cannot be explained merely on 
mathematical grounds. A priori, there is no reason to assume that the ejection fraction is an explicit function of end-systolic volume index only, almost independent of preload (end-diastolic volume index). Obviously, according to its definition formula, ejection fraction would be exactly linearly related to end-systolic volume index only if end-diastolic volume index is constant. Since end-systolic and end-diastolic volume indices usually vary in an unpredictable manner, the relation found is not obvious, and requires the involvement of a regulatory mechanism, which, at least partially, possibly resides in the sympathetic nervous system. This explanation follows from the decreased correlation between the ejection fraction and end-systolic volume index observed in our group of patients under chronic adrenergic blockade, as well as from information ${ }^{916}$ derived from denervated hearts, where we noticed that the relation between the ejection fraction and endsystolic volume index was virtually absent. Perhaps the mechanism for relating the ejection fraction to end-systolic volume index originates from the synchrony of contraction, which, for example, is enhanced during sympathetic stimulation of the heart as shown by Randall and Kelso. ${ }^{17}$

Elsewhere, ${ }^{916}$ we have collected ample evidence that the end-systolic volume index is a more basic index of cardiac performance: it is the final determinant of the pressure-volume loop once pre- and afterload conditions are given; it characterises the left ventricular function curve, and it is the key variable of the systolic elastance index. Our notion concerning the importance of the end-systolic volume index is supported by the study of Borow et al.,$^{18}$ who inferred that it reflects myocardial contractility, and showed that in patients with valvular regurgitation it was a useful predictor of mortality and postoperative left ventricular function. These investigators also noted that neither preoperative end-diastolic volume index nor ejection fraction were statistically significant predictors, in contrast to the end-systolic volume index. In addition, other investigators ${ }^{812}$ have emphasised the prominent role of end-systolic volume index in the analysis of left ventricular performance. The above arguments, along with the documented importance ${ }^{1012}$ of systolic elastance indices, enhance the crucial role played by end-systolic volume for the determination of the performance of the heart. The high correlation between the ejection fraction and end-systolic volume index documented in this study suggests that both variables are not only mathematically, but also functionally interconnected, if indeed the beta-adrenergic system is operating normally.

Since, in the past, no rationale other than empirical observations has been provided to promote ejection fraction as an index of pump performance, it is conceivable that the applicability of the ejection fraction in fact stems from this simple relation between the ejection fraction and end-systolic volume index. The lower correlation found after adrenergic blockade may also explain the inadequacy ${ }^{3}$ of the ejection fraction to judge left ventricular performance under such circumstances, as well as being the clue to the current controversy ${ }^{48}$ " 1 as to whether ejection fraction is a universal and unambiguous index of cardiac performance.

Consequently, it is necessary to scrutinise the relative importance of the ejection fraction and end-systolic volume index, especially in cases when the relation between the two variables is not precisely defined, as for example after beta-blockade or after denervation.

Beta-blockers are widely used, for example, in patients with classic angina, with an estimated abundance of 70 to $80 \%$ according to a recent review, ${ }^{19}$ and $69 \%$ in our patient group described here. This study indicates that these drugs induce a significant dissociation of the connection between ejection fraction and end-systolic volume index. Consequently, it is important to focus attention on end-systolic volume index ${ }^{818}$ and related indices ${ }^{9-12}$ of cardiac performance, rather than on the derived variable ejection fraction as was done in the past during volumetric analysis of left ventricular function in patients, including those under beta-adrenergic blockade.

We thank Dr R W Brower and J de Hooge for data base management, as well as José Koers for assistance.

\section{References}

1 Cohn PF, Gorlin R, Cohn LH, Collins JJ Jr. Left ventricular ejection fraction as a prognostic guide in surgical treatment of coronary and valvular heart disease. $A m \mathcal{F}$ Cardiol 1974; 34: 136-41.

2 Linhart JW, Mintz GS, Segal BL, Kawai N, Kotler MN. Left ventricular volume measurement by echocardiography: fact or fiction? Am $\mathcal{F}$ Cardiol 1975; 36: 114-8.

3 Karliner JS, Gault JH, Bouchard RJ, Holzer J. Factors influencing the ejection fraction and the mean rate of circumferential fibre shortening during atrial fibrillation in man. Cardiovasc Res 1974; 8: 18-25.

4 Krayenbühl HP, Bussman WD, Turina M, Lüthy E. Is the ejection fraction an index of myocardial contractility? Cardiologia 1968; 53: 1-10.

5 Feild BJ, Russell RO, Jr, Moraski RE, et al. Left ventricular size and function and heart size in the year following myocardial infarction. Circulation 1974; 50: 331-9.

6 Sharma B, Goodwin JF, Raphael MJ, Steiner RE, Rainbow RG, Taylor SH. Left ventricular angiography on exercise: a new method of assessing left ventricular function in ischaemic heart disease. Br Heart $\mathcal{F} 1976$; 38: 59-70.

7 Rigaud M, Rocha P, Boschat J, Farcot JC, Bardet J, Bourdarias JP. Regional left ventricular function assessed 
by contrast angiography in acute myocardial infarction. Circulation 1979; 60: 130-9.

8 Mitchell JH, Wildenthal K. Analysis of left ventricular function. Proc $R$ Soc Med 1972; 65: 542-5.

9 Kerkhof PLM. Computer analysis of the relation between ejection fraction and ventricular volume, with special emphasis on the role of end-systolic volume. Automedica 1980; 3: 207-44.

10 Sagawa K, Suga H, Shoukas AA, Bakalar KM. Endsystolic pressure/volume ratio: a new index of ventricular contractility. Am f Cardiol 1977; 40: 748-53.

11 Kerkhof PLM, Van Dijk AD, Aouw Jong TT, Koops J, Moene RJ, Baan J. Pump function of the left ventricle evaluated from pressure-volume loops. In Baan J, Arntzenius AC, Yellin EL, eds. Cardiac dymamics. The Hague: Martinus Nijhoff, 1980: 279-91.

12 Grossman W, Braunwald E, Mann T, McLaurin LP, Green LH. Contractile state of the left ventricle in man as evaluated from end-systolic pressure-volume relations. Circulation 1977; 56: 845-52.

13 Dodge HT. Determination of left ventricular volume and mass. Radiol Clin North Am 1971; 9: 459-67.

14 Lange PE, Onnasch D, Farr FL, Heintzen PH. Angiocardiographic left ventricular volume determination.
Accuracy, as determined from human casts, and clinical application. Eur $\mathcal{F}$ Cardiol 1978; 8: 449-76.

15 Bristow JD, Van Zee BB, Judkins MP. Systolic and diastolic abnormalities of the left ventricle in coronary artery disease. Circulation 1970; 42: 219-28.

16 Kerkhof PLM, van der Velde ET, van Dijk AD, Koops J, Baan J. Can sarcomere dynamics explain the systolic behavior of the left ventricle? In: Kenner Th, ed. Cardiovascular system dynamics: models and measurements. New York: Plenum Press, 1981: (in press).

17 Randall WC, Kelso AF. Dynamic basis for sympathetic cardiac augmentation. Am f Physiol 1960; 198: 971-4.

18 Borow KM, Green LH, Mann T, et al. End-systolic volume as a predictor of postoperative left ventricular performance in volume overload from valvular regurgitation. Am F Med 1980; 68: 655-63.

19 Opie LH. Drugs and the heart. part I: Beta-blocking agents. Lancet 1980; i: 693-8.

Requests for reprints to Dr P L M Kerkhof, Department of Veterinary Physiology, State University Utrecht, A. Numankade 93, 3572 KW Utrecht, The Netherlands. 